
\title{
is Research Suare \\ Left Ventricular Strain and Left Atrial Strain Are Impaired During Hemodialysis in Children
}

\section{Tam T Doan ( $\nabla$ tam.doan@bcm.edu )}

Baylor College of Medicine https://orcid.org/0000-0002-8256-1535

\section{Poyyapakkam Srivaths}

Baylor College of Medicine

Asela Liu

Baylor College of Medicine

J. Kevin Wilkes

Baylor College of Medicine

Alexandra Idrovo

Children's Hospital of The King's Daughters

\section{Ayse Akcan-Arikan}

Baylor College of Medicine

\section{Sarah Swartz}

Baylor College of Medicine

\section{Jessica Geer}

Baylor College of Medicine

\section{Carolyn A. Altman}

Baylor College of Medicine

\section{Nancy A. Ayres}

Baylor College of Medicine

\section{Robert W. Loar}

Cook Children's Medical Center

\section{Ricardo Pignatelli}

Baylor College of Medicine

\section{Research Article}

Keywords: Pediatric Hemodialysis, Speckle Tracking Echocardiography, Mechanical Dispersion Index, Diastolic Strain, Left Atrial Strain

Posted Date: April 13th, 2021

DOl: https://doi.org/10.21203/rs.3.rs-311082/v2

License: @ (i) This work is licensed under a Creative Commons Attribution 4.0 International License. Read Full License 
Version of Record: A version of this preprint was published at The International Journal of Cardiovascular Imaging on July 21st, 2021. See the published version at https://doi.org/10.1007/s10554-021-02350-9. 


\section{Abstract \\ Purpose}

We aimed to investigate intradialytic changes in ventricular and atrial function using speckle tracking echocardiography (STE) in pediatric hemodialysis (HD).

\section{Methods}

Children with HD vintage > 3 months were enrolled, and echocardiography was performed prior to, during, and after HD. STE was analyzed using GE EchoPAC. Left ventricular (LV) global longitudinal strain (GLS), strain rate $(\mathrm{Sr})$, and mechanical dispersion index (MDI) were calculated as the average from 3 apical views; diastolic strain (Ds) and Sr from 4-chamber tracing; left atrial strain (LAS) and Sr from the 4- and 2-chamber views.

\section{Results}

A total of 15 patients were enrolled at a median age of 12 years (IQR 8,16$)$ and median HD vintage of 13 months (IQR 9, 25). GLS worsened during HD $(-15.8 \pm 2.2 \%$ vs $-19.9 \pm 1.9 \%, \mathrm{p}<0.001)$. Post-HD GLS was associated with BP decrease (coefficient $=0.62, p=0.01$ ). LV MDI and systolic Sr did not change. LV Ds progressively worsened $(-8.4 \%(-9.2,-8.0)$ vs $-11.9 \%(-13.4,-10.3), p<0.001)$. LAS changes at mid-HD returned to baseline post-HD. Ds, DSr, LAS, LASr were not associated with BV removal or BP decrease $(p>0.1)$.

\section{Conclusions}

Intradialytic LV strain and LAS changes consistent with subclinical systolic and diastolic dysfunction were observed during HD in children. Changes in Ds, DSr, LAS, and LASr were not associated with BP change or BV removal and may be related to the disease progression. Longitudinal study using these novel indices may unfold the effect of these subclinical changes on long-term cardiovascular health in children requiring chronic HD.

\section{Introduction}

Cardiovascular complications are common among children with end-stage kidney disease (ESKD) with up to 30\% of deaths in children on hemodialysis (HD) due to cardiovascular events [1, 2]. Left ventricular (LV) global longitudinal strain (GLS), mechanical dispersion index (MDI), and the ratio of early mitral inflow velocity $(E)$ to global diastolic strain rate (Sr) in early diastole (E/DSrE) using 2-dimensional (2-D) speckle tracking echocardiography (STE) are predictors of ventricular arrhythmia and sudden cardiac death in adult dialysis patients $[3,4]$

Data on cardiac strain imaging in children with ESRD and HD are limited to markers of systolic function [5-7]. In children on dialysis, retrospective longitudinal studies demonstrated impaired GLS improved after kidney transplantation $[5,6]$. Published literature on the assessment of diastolic function in this scenario has been limited to spectral and tissue Doppler imaging, with one study showing diastolic dysfunction at all stages including post transplantation [6]. Markers of diastolic function and left atrial strain (LAS) using 2-D STE can 
predict outcomes and be potentially better than spectral and tissue Doppler imaging in adults with certain disease states [8]. There is scant data regarding changes in systolic and diastolic function during HD in children.

In this secondary analysis of data from a prospective study of GLS and cerebral oxygenation, we focused on LV mechanical dispersion index (MDI), systolic Sr, Ds and Sr, and LAS using 2-D STE during HD in children on chronic HD for ESKD. We hypothesized that there would be significant intradialytic derangements in MDI, Ds, and LAS when compared to baseline.

\section{Material And Methods}

\section{Study Population}

A total of 15 patients $\leq 20$ years old with ESKD and structurally normal heart, who had been on HD $\geq 3$ months (HD vintage) were enrolled. The study subjects were initially recruited for analysis of GLS and cerebral oxygenation in the form of non-invasive near infrared spectroscopy [9]. Consent was obtained and the study was approved by the Institutional Review Board.

\section{Monitoring and Measurements During Hemodialysis}

Patients' heart rates and blood pressures (BP) were measured every 15 minutes during the HD session. Elevated $\mathrm{BP}$ was determined as systolic and/or diastolic $\mathrm{BP}^{3} 95^{\text {th }}$ percentile for age, sex, and height [10]. Patients were monitored for dialysis-associated morbidity symptoms (lightheadedness, muscle cramps, vomiting, fatigue, lethargy, confusion). Crit-line III TQA (HemaMetrics MDSS GmbH, Germany) was used to assess changes in blood volume (BV). Hemodialysis was performed following standard of care linear fluid removal and stable HD prescription with typical dialysate baths including calcium and potassium.

\section{Echocardiography}

Transthoracic echocardiography was performed at the beginning of HD (pre-HD), 2 hours later (mid-HD), and at the end of a 4-hour HD (post-HD) by a trained research pediatric cardiac sonographer (A.N.) using a commercially available ultrasound system (GE Vivid E95 Ultrasound System, GE Medical Systems, Milwaukee, WI). Apical 4chamber, 2-chamber, and 3-chamber views were obtained, each cine image included three cardiac cycles, settings were adjusted manually to obtain frame rates of 50-70 frames/second, and the left atrium was included for LAS analyses. Left ventricular ejection fraction (LVEF) was calculated using bi-plane Simpson's method, and was considered normal when $\geq 55 \%$ [11]. Left ventricular end-diastolic and end-systolic dimensions were measured on a 2-D short axis and $z$ scores were derived based on data from the Pediatric Heart Network [12]. Spectral Doppler assessment of the mitral inflow was obtained at each HD stage and peak velocities of flow were recorded during early $(\mathrm{E})$ and late diastole $(\mathrm{A})$. Pulsed wave tissue Doppler imaging was used to measure early diastolic myocardial relaxation velocity from the lateral mitral annulus (e'), and E/e' ratio was derived.

\section{Speckle Tracking Analysis}

Echocardiographic images were sent to a single software station and strain analyses were performed offline (EchoPAC Clinical Workstation Software, GE Vingmed Ultrasound). Three sets of images were available for each study subject for analysis of changes at mid-HD and post-HD compared to pre-HD. 
Myocardial tracking was assessed for each segment and tracing was manually adjusted if necessary. Left ventricular GLS was the average of peak systolic longitudinal strain from the three apical views and was calculated by the software. LV systolic Sr represented the rate of LV deformation in systole. LV GLS and Sr were presented as negative values, which represented the percentage of myocardial shortening from its original length and rate of LV shortening. Impaired GLS was defined as GLS (negative value) > the upper limit of normal value for age [13]. Time to peak systolic longitudinal strain was measured from the onset of QRS on ECG to peak systolic strain. Left ventricular MDI was measured automatically by the system from the GLS curves as the standard deviation of time to peak longitudinal strain of all 17 LV segments marking the temporal difference of LV deformation [14].

Left ventricular global longitudinal diastolic strain (Ds) and diastolic Sr during early diastole (DSrE) and late diastole (DSrA) were measured from the apical 4-chamber view. Time from peak R wave of the QRS complex to peak $E$ wave was measured and used to identify Ds at the time of peak E wave. Ratio of E/Ds, E/DSrE, and E/DSrA were calculated as additional markers of diastolic function.

Left atrial strain analysis was performed using dedicated images with good definition of the left atrial wall on the 4-chamber and 2-chamber views. The left atrial appendage and entrance of the pulmonary veins were excluded from the tracing. The initial up-sloping of the $P$ wave was used as the reference point for LAS. Left atrial strain

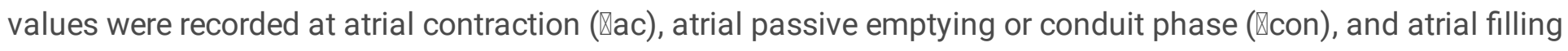

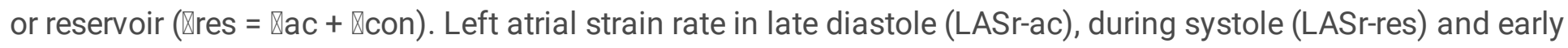
diastole (LASr-con) were measured along the average Sr curve as the parameters of contractile, reservoir, and conduit function, respectively. The reported LAS and LASr values are the average of the 4- and 2-chamber values.

\section{Statistical Analysis}

Statistical analysis was performed using Stata ${ }^{\mathrm{TM}} 14.2$ (StataCorp LP, College Station, TX). Variates were presented as mean \pm standard deviation, median and $25-75 \%$ interquartile ranges (IQR), and number and percentage, where appropriate. Normality of continuous variables was assessed by frequency distribution (histogram) and Shapiro-Wilk test. Variable changes during HD (pre-HD vs mid-HD, pre-HD vs post-HD, and midHD vs post-HD) were compared using paired T test (parametric continuous variables), Wilcoxon signed rank test (non-parametric continuous variables), or McNemar test (paired nominal variables) where appropriate. Simple linear regression analyses were performed to study the association between BV change and BP change and GLS, systolic Sr, MDI, Ds, diastolic Sr, and LAS and Sr at mid-HD and post-HD. A 2-tail p-value of $<0.05$ was considered statistically significant.

\section{Results}

The 15 study subjects were enrolled at a median age of 12 years (IQR 8, 16), median weight of $30.5 \mathrm{~kg}$ (IQR 23.8, 49), median BMI of $18.4 \mathrm{~kg} / \mathrm{m}^{2}$ (IQR 17.3, 21.7), and median HD vintage of 13 months (IQR 9, 25). All patients tolerated HD well with no symptoms. Additional patient characteristics are described in Table 1.

Table 1. Baseline characteristics 


\begin{tabular}{|l|r|}
\hline Demographic Features (N = 15) & $\mathrm{n}(\%)$ \\
\hline Male gender & $9(60)$ \\
\hline Race and ethnicity & $8(53)$ \\
Hispanic & $6(40)$ \\
Black or African American & $1(7)$ \\
Others & \\
\hline Underlying diagnosis & $9(60)$ \\
Congenital anomalies & $4(27)$ \\
Glomerulonephritis & $2(13)$ \\
Other & $10(66.7)$ \\
\hline Elevated blood pressure at baseline $\left(\square 95^{\text {th }}\right.$ percentile) & $3(20)$ \\
Systolic & $1(6.7)$ \\
Diastolic & $6(40)$ \\
Both systolic and diastolic & \\
\hline
\end{tabular}

\section{Hemodynamic Changes During Hemodialysis}

Fluid was steadily removed during HD with a total BV decrease of $-13.7 \pm 3.3 \%$ from baseline with an average actual weight decrease of $2.5 \pm 2.5 \mathrm{~kg}(6.0 \pm 7.2 \%)$. Intradialytic changes in BP and heart rates are shown in Table 2. No patient had symptomatic hypotension requiring intervention.

Table 2. Changes in blood volume and hemodynamics during hemodialysis

\begin{tabular}{l|c|c|c|c|c|c|}
\hline Jarameters & Pre-HD & Mid-HD & Post-HD & P (pre-mid) & P (mid-post) & P (pre-post) \\
\hline 3V change, \% & 0 & $9.2 \pm 1.9$ & $13.7 \pm 3.3$ & $<0.001$ & $<0.001$ & $<0.001$ \\
\hline Jeart rate, bpm & $85(78,87)$ & $90(80,102)$ & $98(85,111)$ & 0.02 & 0.4 & 0.005 \\
\hline Jystolic BP, mmHg & $126 \pm 20$ & $117 \pm 15$ & $109 \pm 11$ & 0.01 & $<0.05$ & 0.003 \\
\hline Jiastolic BP, mmHg & $76 \pm 11$ & $72 \pm 10$ & $69 \pm 10$ & $<0.05$ & 0.1 & 0.001 \\
\hline Mean BP, mmHg & $80 \pm 11$ & $75 \pm 9$ & $71 \pm 8$ & 0.005 & 0.04 & $<0.001$ \\
\hline Zlevated BP, n (\%) & $10(66.7)$ & $7(46.7)$ & $5(33.3)$ & 0.5 & 0.3 & 0.06 \\
\hline
\end{tabular}

$\mathrm{BV}=$ blood volume; $\mathrm{bpm}=$ beats per minute; $\mathrm{BP}=$ blood pressure 
Two-dimensional, Spectral Doppler, and Speckle Tracking Echocardiography

Changes in LV dimensions, LVEF, spectral and tissue Doppler are depicted in Table 3.

The LV GLS was impaired at mid-HD (3.3 $\pm 2.4 \%)$ and post-HD ( $4.0 \pm 2.4 \%)$ compared to baseline, while no significant change in MDI or systolic Sr was detected during HD (Table 4). The LV Ds progressively worsened, totaling $3.3 \pm 1.8 \%$ from baseline. No significant change to other diastolic strain parameters (E/Ds, E/DSrE, DSrA, E/DSrA) was noted. The individual changes of LV GLS, MPI, Ds, DSrE, ロres and Sr-res during HD are depicted on Figure 1.

Table 3. Intradialytic changes in LV size, LVEF, spectral and tissue Doppler imaging parameters.

\begin{tabular}{|c|c|c|c|c|c|c|}
\hline arameters & Pre-HD (IQR) & Mid-HD (IQR) & Post-HD (IQR) & P (pre-mid) & P (mid-post) & $\mathrm{P}$ (pre-post) \\
\hline VEDD, mm & $40.8 \pm 0.8$ & $36.2 \pm 0.7$ & $33.7 \pm 0.6$ & $<0.001$ & 0.001 & $<0.001$ \\
\hline VEDD z score & $-0.5(-1.4,0.0)$ & $-1.6(-2.7,-0.6)$ & $-2.7(-2.9,-1.7)$ & 0.001 & $<0.001$ & $<0.001$ \\
\hline VESD, mm & $28.3 \pm 0.6$ & $25.9 \pm 0.5$ & $24.5 \pm 0.5$ & $<0.001$ & 0.003 & $<0.001$ \\
\hline VEF, \% & $63 \pm 3$ & $61 \pm 4$ & $60 \pm 3$ & $<0.001$ & 0.1 & $<0.001$ \\
\hline VEF ૧ 55\%, n (\%) & $15(100)$ & $15(100)$ & $15(100)$ & & & \\
\hline velocity, $\mathrm{cm} / \mathrm{s}$ & $98 \pm 19$ & $71 \pm 14$ & $73 \pm 18$ & $<0.001$ & 0.7 & $<0.001$ \\
\hline velocity, $\mathrm{cm} / \mathrm{s}$ & $55(49,65)$ & $51(43,55)$ & $48(39,61)$ & 0.09 & 0.6 & 0.06 \\
\hline /A ratio & $1.8(1.4,2.1)$ & $1.3(1.2,1.7)$ & $1.4(1.2,1.8)$ & 0.01 & 0.5 & 0.04 \\
\hline /e' (lateral) & $6.5(4.7,7.1)$ & $6.0(4.5,8.7)$ & $5.6(4.6,6.9)$ & 0.5 & 0.6 & 0.2 \\
\hline
\end{tabular}

LVEDD = left ventricular end-diastolic dimension; LVESD = left ventricular end-systolic dimension; LVEF = left ventricular ejection fraction.

Table 4. Intradialytic changes in LV systolic and diastolic strain, and LAS 


\begin{tabular}{|c|c|c|c|c|c|c|}
\hline ımeters & Pre-HD (IQR) & Mid-HD (IQR) & Post-HD (IQR) & $\begin{array}{c}\text { P (pre- } \\
\text { mid) }\end{array}$ & $\begin{array}{l}\text { P (mid- } \\
\text { post) }\end{array}$ & $\begin{array}{l}\text { P (pre- } \\
\text { post) }\end{array}$ \\
\hline iLS, \% & $-19.9 \pm 1.9$ & $-16.5 \pm 2.3$ & $-15.8 \pm 2.2$ & $<0.001$ & 0.1 & $<0.001$ \\
\hline aired GLS,[13] n & $8(53.3)$ & $15(100)$ & $15(100)$ & & & \\
\hline ;r, \% & $-1.0(-1.1,-0.9)$ & $-1.0(-1.2,-0.8)$ & $-0.9(-1.0,-0.8)$ & 0.5 & 0.2 & 0.09 \\
\hline msec & $36 \pm 9$ & $38 \pm 11$ & $37 \pm 9$ & 0.5 & 0.3 & 0.5 \\
\hline ) $\%$ & $\begin{array}{c}-11.9(-13.4 \\
-10.3)\end{array}$ & $\begin{array}{c}-9.8(-10.9 \\
-8.8)\end{array}$ & $-8.4(-9.2,-8.0)$ & 0.004 & 0.04 & $<0.001$ \\
\hline ; $(\mathrm{cm} / \mathrm{s}) / \%$ & $-7.5(-10.0,-6.9)$ & $-6.8(-9.7,-6.0)$ & $-8.1(-10.0,-6.5)$ & 0.07 & 0.08 & 1 \\
\hline$\Xi, \%$ & $1.8(1.7,2.0)$ & $1.3(1.0,1.6)$ & $1.2(1.0,1.6)$ & 0.01 & 0.1 & $<0.001$ \\
\hline $\operatorname{srE},(\mathrm{cm} / \mathrm{s}) / \%$ & $55(40,66)$ & $53(41,65)$ & $56(47,72)$ & 0.6 & 0.08 & 0.5 \\
\hline A, \% & $0.6(0.5,0.7)$ & $0.5(0.3,0.8)$ & $0.4(0.4,0.5)$ & 0.3 & 0.5 & 0.1 \\
\hline $\operatorname{srA},(\mathrm{cm} / \mathrm{s}) / \%$ & $163(146,182)$ & $157(100,185)$ & $197(136,210)$ & 0.5 & 0.7 & 0.9 \\
\hline$\%$ & $-13.6(-17.1,-9.1)$ & $\begin{array}{c}-10(-19.7 \\
-7.1)\end{array}$ & $\begin{array}{c}-11.5(-19.7 \\
-8.3)\end{array}$ & 0.6 & 0.3 & 0.9 \\
\hline$\%$ & $22.6 \pm 8.6$ & $15.3 \pm 8.3$ & $18.5 \pm 10.0$ & 0.04 & 0.3 & 0.1 \\
\hline$\%$ & $36.9 \pm 6.2$ & $28.3 \pm 5.4$ & $32.9 \pm 8.4$ & 0.001 & 0.1 & 0.06 \\
\hline es, $(\mathrm{cm} / \mathrm{s}) / \%$ & $237 \pm 18$ & $337 \pm 22$ & $315 \pm 30$ & 0.001 & 0.5 & 0.009 \\
\hline ;r-ac, \% & $-1.6(-2.0,-1.1)$ & $-1.4(-2.2,-1.2)$ & $-1.7(-2.2,-1.2)$ & 0.9 & 0.1 & 0.6 \\
\hline ir-con, \% & $1.6(1.3,1.8)$ & $1.3(1.1,1.7)$ & $1.6(1.3,1.8)$ & $<0.005$ & 0.04 & 0.1 \\
\hline ;r-res, \% & $-2.7(-2.9,-2.1)$ & $-1.9(-2.3,-1.4)$ & $-2.1(-3.0,-1.8)$ & 0.004 & 0.04 & 0.1 \\
\hline
\end{tabular}


GLS = global longitudinal strain; $\mathrm{Sr}=$ strain rate; $\mathrm{MDI}=$ mechanical dispersion index; $\mathrm{Ds}=$ diastolic strain; $\mathrm{LA}=$ left atrial; $\square=$ strain; ac $=$ atrial contraction; con = atrial passive emptying or conduit; res = atrial filling or reservoir Intradialytic STE Changes \& BV and Mean BP Changes

There is a moderate correlation between mean BP decrease and post-HD GLS (coefficient $=0.62, \mathrm{p}=$ 0.01). The correlation between total BV removal and post-HD GLS was not statistically significant (coefficient $=0.49, \mathrm{p}=$ 0.06) on simple linear regression analyses. Less mean BP reduction was associated with less post-HD GLS impairment (Figure 2).

The mid-HD GLS or intradialytic GLS change from pre-HD to mid-HD or changes in LV global Ds, DSrE, E/DSrE, LAS $\square$ res, Sr-res, E/ロres, \con, and Sr-con at mid-HD and post-HD were not associated with BV change or BP change. Age and gender were not associated with intradialytic STE changes.

\section{Discussion}

In this analysis of children undergoing chronic HD for ESKD with the typical range of underlying congenital and acquired kidney conditions, we observed intradialytic changes in both systolic and diastolic function using novel STE markers. Both mid-HD and post-HD GLS significantly impaired from baseline. Progressive impairment in diastolic function was detected in terms of LV Ds at all three time points. Mid-HD LAS and Sr were impaired from baseline and both LAS and Sr returned to baseline at the end of HD.

\section{Systolic Function}

Similar to other studies, LVEF remained normal ( ${ }^{3} 55 \%$ ) throughout HD in all of our patients. Prior studies in children also showed preserved LVEF when compared to healthy control and nonsignificant LVEF change during HD $[5,6]$. Baseline GLS in children with ESKD and HD have been noted to be mildly impaired in 2 separate previous studies $(-19.7 \pm 2.8 \%[6]$ and $-20.31 \pm 3.58 \%[5])$, which reported GLS similar to the baseline GLS in our study $(-19.9 \pm 1.9 \%)$. In our study, 53\% had abnormal GLS at baseline, which worsened during HD with $100 \%$ having abnormal GLS at mid-HD and post-HD. Post-HD GLS was associated with the degree of decrease in mean BP during HD with greater decrease in BP associated with more significant post-HD GLS impairment. The asociation between post-HD GLS and BV removal was not statistically significant.

Intradialytic LV MDI change was not significant in our study. Predialysis and dialysis adult patients with ventricular arrhythmia or sudden cardiac death had larger MDI 66 (55-74) vs $52(43-65)$ msec $(p=0.04)$ with nonsignificant vintage difference between the 2 groups [15]. Our patients' median HD vintage of 13 months (IQR 9 , 25) was not different from what was reported in this study [15]. In our study, MDI measured $36 \pm 9$ vs $37 \pm 9$ msec after HD, which were lower compared to data in adults [15]. The lower MDI values in our study population likely indicates the lack of LV dyssynchrony. We hypothesized the difference in MDI between pediatric patients and adults were related to underlying etiologies and fewer comorbidities in children.

Left ventricular GLS was the only marker of systolic function that demonstrated intradialytic impairment in our study. The impaired GLS would not have been detected by conventional echo imaging as LVEF remained normal, 
indicating that more sophisticated noninvasive imaging techniques might be paramount to detect adverse impact of HD in children which likely occurs repetitively with each HD session in children.

\section{Diastolic Function}

Diastolic dysfunction diagnosed by spectral and tissue Doppler imaging has been reported in children with ESKD requiring long-term $\mathrm{HD}[6,16,17]$. Diastolic dysfunction developed early in the course of children with chronic kidney disease and was present up to 18 months after renal transplantation [6]. We observed progressive intradialytic Ds and DSrE impairment which was not associated with BV removal or BP change. This progressive intradialytic ventricular diastolic changes are likely repetitive and potentially contributory to diastolic dysfunction in patients on chronic HD for ESKD, which may persist after transplantation [6]. Evolving data in adults showed asymptomatic diastolic dysfunction is associated with subsequent heart failure and mortality; addressing these preclinical changes and echocardiographic surveillance may delay symptomatic progression and improve outcome [17]. Longitudinal studies are necessary to understand how diastolic function plays a role in cardiovascular outcome of children who are on chronic HD.

\section{Left Atrial Strain}

Left atrial strain reservoir has been identified as a new emerging reliable marker of diastolic dysfunction in adults; it better reflects instantaneous left atrial pressure with greater prognostic value than left atrial volume index [18]. Left atrial strain is a sensitive marker of early diastolic dysfunction, linearly worsens to the progression of diastolic dysfunction, and improves as LV filling pressure improves [19]. While the use of LAS has been studied in HD adults and found to be predictive of outcome [20], we found no prior study assessing LAS in pediatric chronic HD. In our study, LAS and Sr during the atrial early emptying (conduit) and atrial filling (reservoir) were impaired at mid-HD but returned to baseline at the end of HD. When combined with mitral inflow E velocity, the E/囚res increased at mid-HD and remained increased at post-HD. The E/\res ratio has been identified as a useful parameter in the prediction of total and cardiovascular mortality in adult HD patients [20]. Similar to Ds, these LAS and Sr changes in our study were not associated with BV removal or BP change. Left atrial contractile function changes during HD using tissue velocity and Sr during late diastole were noted to be preload-independent in 41 adult HD patients, which was similar to our observation in children in this study [21]. Further studies are needed to understand the role of these intradialytic changes in the progression of diastolic dysfunction in children with chronic HD.

Children with ESKD are at risk of cardiovascular health issues, possibly related to their disease process, complications of the disease, and treatment which can lead to microvascular dysfunction [13]. Our study results demonstrated progressive intradialytic LV diastolic dysfunction, which was not associated with the degree of fluid removal or BP change. It may have occurred as a natural history of the disease process. Addressing vascular dysfunction is valuable in adults to reduce long-term major adverse cardiovascular complications [13]. Our results are not sufficient to postulate pathophysiology related to cardiovascular complications seen in this study population. However, they may serve as a proof that these novel tools can be utilized to measure the magnitude of subclinical function changes and future research as we strive to understand the disease process, minimize harm, and improve the care and outcome of children with ESKD requiring HD.

Our study has limitations of a single center with small sample size. We were only able to assess patients during one HD treatment and did not follow them longitudinally post treatment to assess whether the observed 
abnormalities resolve during the recovery period after HD. We did not have access to real time hemodynamic indices such as continuous BP or cardiac output monitoring. However, none of our patients had overt cardiovascular risk factors, all had uncomplicated asymptomatic treatments, and relatively short vintage, underscoring the adverse hemodynamic burden of HD on organ function.

\section{Conclusions}

Significant impairment in STE markers of systolic and diastolic function, including LAS, were observed during HD in children. Intradialytic changes in systolic GLS were associated with BP change whereas changes in Ds, diastolic Sr, and LAS were not associated with BP change or BV removal. Longitudinal assessment using these novel non-invasive indices may unfold the effect of potentially repetitive and cumulative subclinical changes on long-term cardiovascular health in children with ESKD who are on chronic HD.

\section{Declarations}

Funding: This research did not receive any specific grant from funding agencies in the public, commercial, or notfor-profit sectors

Conflicts of interest/Competing interests: The authors do not have conflicts of interest or competing interests.

Availability of data and material: Data are available upon request.

Code availability: Not applicable

Authors' contributions: All authors contributed to the study conception and design. Material preparation, data collection and analysis were performed by all authors, Tam T. Doan, Poyyapakkam Srivaths, Asela Liu, J. Kevin Wilkes, Alexandra Idrovo, Ayse Akcan-Arikan, Sarah Swartz, Jessica Geer, Carolyn A. Altman, Nancy A. Ayres, Robert W. Loar, and Ricardo Pignatelli. The first draft of the manuscript was written by Tam T Doan and all authors commented on previous versions of the manuscript. All authors read and approved the final manuscript.

Ethics approval: The primary study was approved by the Institutional Review Board at Baylor College of Medicine. The study was performed in accordance with the ethical standards as laid down in the 1964 Declaration of Helsinki and its later amendments or comparable ethical standards.

Consent to participate Informed consents were obtained at the time of the primary study. No additional consent form was obtained for this secondary analysis.

Consent for publication: Not applicable.

\section{References}

1. Shroff R, Smith C, Ranchin B et al (2019) Effects of hemodiafiltration versus conventional hemodialysis in children with ESKD: The HDF, heart and height study. J Am Soc Nephrol 30:678-691. https://doi.org/10.1681/ASN.2018100990

2. US Renal Data System (2011) USRDS 2011 Annual Data Report. Atlas of Chronic Kidney Disease and EndStage Renal Disease in the United States. National Institutes of Health, National Institute of Diabetes and 
Digestive and Kidney Diseases, Bethesda

3. Huang J-C, Su H-M, Wu P-Y et al (2019) Ratio of Early Mitral Inflow Velocity to the Global Diastolic Strain Rate and Global Left Ventricular Longitudinal Systolic Strain Predict Overall Mortality and Major Adverse Cardiovascular Events in Hemodialysis Patients. Dis Markers 2019:Article ID 7512805. https://doi.org/10.1155/2019/7512805

4. Hensen LCR, Goossens K, Delgado V et al (2018) Prevalence of left ventricular systolic dysfunction in predialysis and dialysis patients with preserved left ventricular ejection fraction. Eur J Heart Fail 20:560-568. https://doi.org/10.1002/ejhf.1077

5. Rakha S, Hafez M, Bakr A, Hamdy N (2020) Changes of cardiac functions after hemodialysis session in pediatric patients with end-stage renal disease: conventional echocardiography and two-dimensional speckle tracking study. Pediatr Nephrol 35:861-870. https://doi.org/10.1007/s00467-019-04460-y

6. Rumman RK, Ramroop R, Chanchlani R et al (2017) Longitudinal assessment of myocardial function in childhood chronic kidney disease, during dialysis, and following kidney transplantation. Pediatr Nephrol 32:1401-1410. https://doi.org/10.1007/s00467-017-3622-7

7. Chinali M, Matteucci MC, Franceschini A et al (2015) Advanced parameters of cardiac mechanics in children with CKD: The 4C study. Clin J Am Soc Nephrol 10:1357-1363. https://doi.org/10.2215/CJN.10921114

8. Nagueh SF, Smiseth OA, Appleton CP et al (2016) Recommendations for the Evaluation of Left Ventricular Diastolic Function by Echocardiography: An Update from the American Society of Echocardiography and the European Association of Cardiovascular Imaging. J Am Soc Echocardiogr 29:277-314. https://doi.org/10.1016/j.echo.2016.01.011

9. Idrovo A, Srivaths P, Akcan-Arikan A et al (2020) Worsening myocardial strain with preserved cerebral oxygenation in pediatric chronic hemodialysis. In: Hemodialysis International. p A17

10. Flynn JT, Kaelber DC, Baker-Smith CM et al (2017) Clinical Practice Guideline for Screening and Management of High Blood Pressure in Children and Adolescents. Pediatrics 140:e20171904. https://doi.org/10.1542/peds.2017-1904

11. Lopez L, Colan SD, Frommelt PC et al (2010) Recommendations for Quantification Methods During the Performance of a Pediatric Echocardiogram: A Report From the Pediatric Measurements Writing Group of the American Society of Echocardiography Pediatric and Congenital Heart Disease Council. J Am Soc Echocardiogr 23:465-495. https://doi.org/10.1016/j.echo.2010.03.019

12. Lopez L, Colan S, Stylianou M et al (2017) Relationship of Echocardiographic Z Scores Adjusted for Body Surface Area to Age, Sex, Race, and Ethnicity: The Pediatric Heart Network Normal Echocardiogram Database. Circ Cardiovasc Imaging 10:1-7. https://doi.org/10.1161/CIRCIMAGING.117.006979

13. Levy PT, Machefsky A, Sanchez AA et al (2016) Reference Ranges of Left Ventricular Strain Measures by Two-Dimensional Speckle-Tracking Echocardiography in Children: A Systematic Review and Meta-Analysis. J Am Soc Echocardiogr 29:209-225.e6. https://doi.org/10.1016/j.echo.2015.11.016

14. Aagaard EN, Kvisvik B, Pervez MO et al (2020) Left ventricular mechanical dispersion in a general population: Data from the Akershus Cardiac Examination 1950 study. Eur Heart J Cardiovasc Imaging 21:183-190. https://doi.org/10.1093/ehjci/jez210

15. Hensen LCR, Goossens K, Podlesnikar T et al (2018) Left Ventricular Mechanical Dispersion and Global Longitudinal Strain and Ventricular Arrhythmias in Predialysis and Dialysis Patients. J Am Soc Echocardiogr 31:777-783. https://doi.org/10.1016/j.echo.2018.01.010 
16. Shamszad P, Slesnick TC, Smith EOB et al (2012) Association between left ventricular mass index and cardiac function in pediatric dialysis patients. Pediatr Nephrol 27:835-841. https://doi.org/10.1007/s00467011-2060-1

17. Kosmala W, Marwick TH (2020) Asymptomatic Left Ventricular Diastolic Dysfunction: Predicting Progression to Symptomatic Heart Failure. JACC Cardiovasc Imaging 13:215-227. https://doi.org/10.1016/j.jcmg.2018.10.039

18. Cho GY, Hwang IC (2020) Left Atrial Strain Measurement: A New Normal for Diastolic Assessment? JACC Cardiovasc Imaging 13:2327-2329. https://doi.org/10.1016/j.jcmg.2020.05.014

19. Thomas L, Marwick TH, Popescu BA et al (2019) Left Atrial Structure and Function, and Left Ventricular Diastolic Dysfunction: JACC State-of-the-Art Review. J Am Coll Cardiol 73:1961-1977. https://doi.org/10.1016/j.jacc.2019.01.059

20. Tsai W-C, Lee W-H, Wu P-Y et al (2019) Ratio of Transmitral E Wave Velocity to Left Atrial Strain as a Useful Predictor of Total and Cardiovascular Mortality in Hemodialysis Patients. J Clin Med 9:85. https://doi.org/10.3390/jcm9010085

21. Park CS, Kim YK, Song HC et al (2012) Effect of preload on left atrial function: Evaluated by tissue Doppler and strain imaging. Eur Heart J Cardiovasc Imaging 13:938-947. https://doi.org/10.1093/ehjci/jes069

\section{Figures}




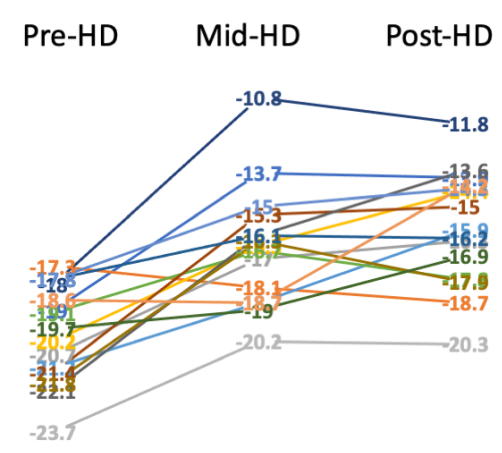

A GLOBAL LONGITUDINAL STRAIN, \%

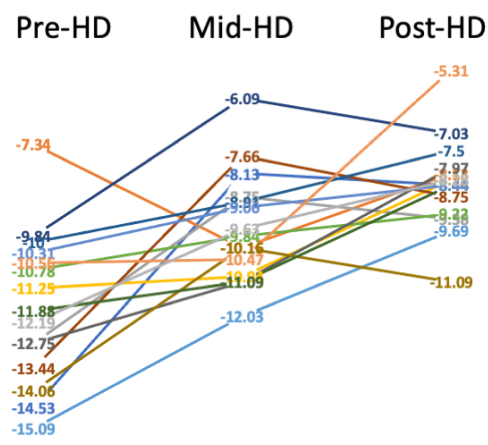

C GLOBAL DIASTOLIC STRAIN, \%

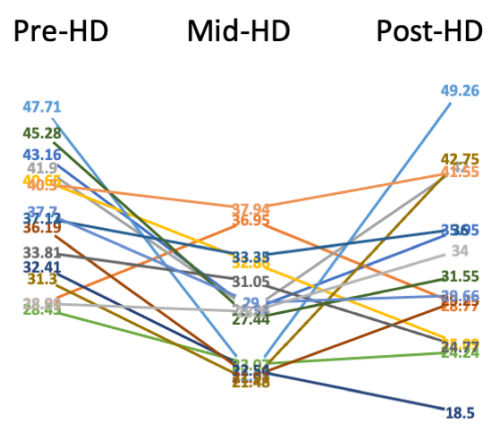

E LEFT ATRIAL STRAIN RESERVOIR, \%
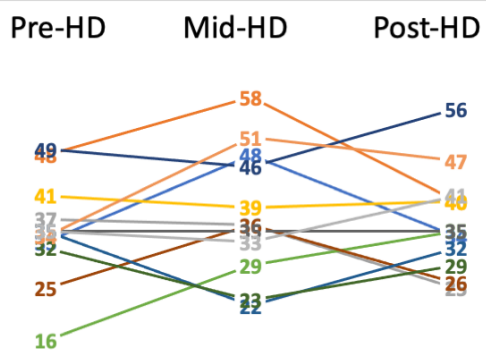

B MECHANICAL DISPERSION INDEX, msec

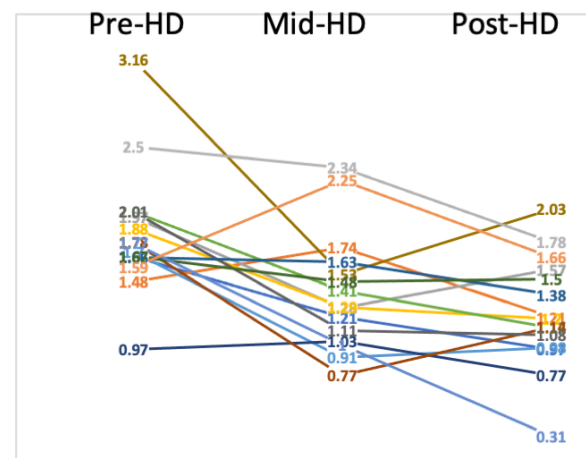

D EARLY DIASTOLIC STRAIN RATE, \%

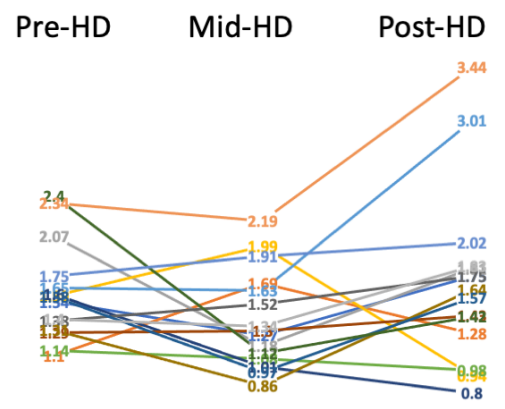

F LEFT ATRIAL STRAIN RATE RESERVOIR,\%

\section{Figure 1}

Trajectory of left ventricular systolic global longitudinal strain (GLS), mechanical dispersion index (MPI), global diastolic strain (Ds), early diastolic strain rate (DSrE), and left atrial strain and strain rate during atrial filling or reservoir (囚res and Sr-res) between pre-HD, mid-HD, and post-HD. 

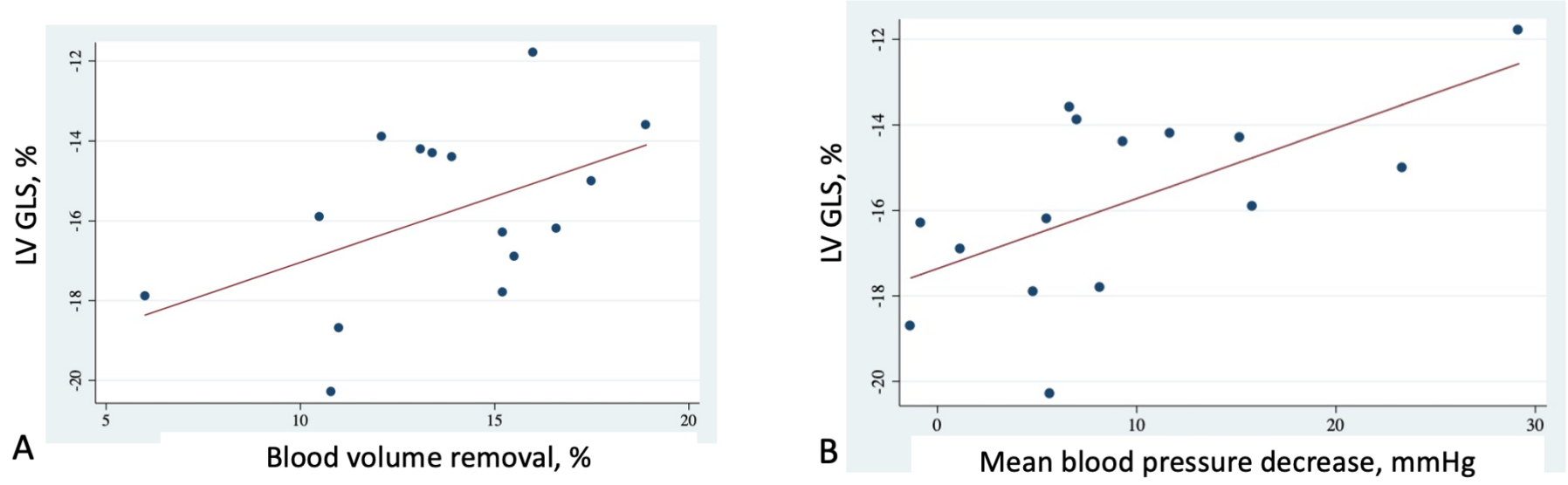

Figure 2

Relationship of total blood volume removal and mean blood pressure decrease with post-HD GLS 